\title{
Anticoagulants and gastrointestinal bleeding
}

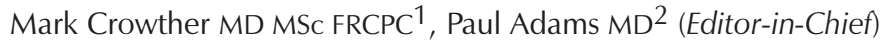

M ark Crowther is an Associate Professor of Medicine at McMaster University Medical Centre. He is a hematologist with a special interest in coagulation.

PA: Mark, with the increasing use of anticoagulants, we are seeing an increase in gastrointestinal (GI) bleeding. While a major GI bleed was formerly considered to be a contraindication to long-term anticoagulation, increasingly, we are being asked by colleagues in cardiology, neurology and other specialties to allow them to continue the anticoagulation despite the recent GI bleeding. As the consultant gastroenterologist, we often have not localized the cause of the bleeding (rectal bleeding), and/or the problem is not focal and immediately amenable to endoscopic therapies (gastritis). Furthermore, the rate of healing of many GI lesions, such as a gastric ulcer, is highly variable. The drugs that are most commonly used are warfarin, clopidogrel, tissue plasminogen activator, acetylsalicylic acid and low molecular weight heparin.

Let's begin with a typical case. A 75-yearold woman is on warfarin for atrial fibrillation and presents with hematemesis. Endoscopy shows a gastric ulcer and diffuse gastritis. Should we stop the warfarin, and for how long?

MC: Major bleeding in patients who are taking warfarin remains the most common toxicity of this therapy. Although controversial, most studies suggest that $1 \%$ to $3 \%$ of patients taking warfarin will present each year with a bleeding episode requiring medical intervention; about one in 10 of these episodes will be fatal. Although intracerebral bleeding is the most feared complication of warfarin with a case-fatality rate of $40 \%$ to $50 \%$, gastrointestinal hemorrhage is the most frequent type of bleeding that prompts medical intervention.

Clinicians routinely overestimate the risk of thrombosis and underestimate the consequences of bleeding when faced with a patient who was taking warfarin and who presents with bleeding. An otherwise well 75-year-old patient has a low annual risk of stroke; certainly, the risk of mortality and major morbidity due to bleeding will exceed that of stroke if warfarin is reintroduced early in this patient. In this particular case, assuming the patient had no additional risk factors for stroke, such as prior stroke/transient ischemic attack, valvular heart disease, left ventricular dysfunction or uncontrolled hypertension, I would leave her off all anticoagulants until adequate healing of the ulcer was demonstrated. In some cases, this might be several months. The patient and their family should be counselled about the risk of stroke; however, once again, the risk of warfarin-associated toxicity, in this particular case, outweighs warfarin-associated benefits.

PA: Does the plan change if the patient has a mechanical heart valve or has just had a stroke?

MC: As patients acquire additional risk factors for stroke, my comfort with extended warfarin withdrawal declines. However, once again, in the immediate peribleed period, and for a period of up to some weeks, the morbidity and mortality associated with warfarin-induced bleeding will exceed the morbidity due to thrombosis.

The risk of thrombosis can also be estimated, which may influence decisions about the duration of warfarin withdrawal. For example, older valves, such as ball-cage or monoleaflet tilting disc valves, appear to be associated with a higher risk of thrombosis, as are valves in the mitral position. Other risk factors for stroke are, in order of importance, prior stroke/transient ischemic attack, left ventricular dysfunction, uncontrolled hypertension or diabetes, and age older than 75 years. For example, a patient with a newer (bileaflet) mechanical aortic valve, normal sinus rhythm and no prior stroke has a low day-over-day risk of thrombosis, whereas a patient with an older mitral position valve and left ventricular dysfunction will have a far higher risk.

When to resume anticoagulants hinges on an estimate of the risk of recurrent bleeding, which, in turn, is predicated on the etiology of the bleed. Thus, in a patient who develops GI bleeding from a reversible (eg, Mallory-Weiss tear) or treatable (eg, peptic ulcer disease not actively bleeding at esophagogastroduodenoscopy) disease, it is reasonable to resume anticoagulants within one to two weeks of the bleed, assuming the patient also receives aggressive treatment for the bleed as required (eg, proton pump inhibition, Helicobacter pylori eradication). On the other hand, if the patient develops GI bleeding from a disease that requires considerable time to resolve (eg, ulcerative colitis, large peptic ulcer disease with bleeding at esophagogastroduodenoscopy), it is reasonable to resume anticoagulants within two to four weeks of the bleed. In such patients, repeat endoscopy may be warranted to ensure disease resolution before anticoagulants are resumed. Finally, the most

${ }_{1}$ Department of Medicine, McMaster University, Hamilton, Ontario; ${ }^{2}$ London Health Sciences Centre, London, Ontario

Correspondence: Dr Mark Crowther, McMaster University, Room L208 St Joseph's Hospital, 50 Charlton Avenue East, Hamilton, Ontario

L8N 4A6. Telephone 905-521-6024, fax 905-540-6568, e-mail crowthrm@mcmaster.ca

Received for publication May 5, 2005. Accepted May 6, 2005 
problematic cases are patients with an irreversible cause of GI bleeding (eg, invasive inoperable neoplasm, grade 3 to 4 esophageal varices), in whom decisions about resuming anticoagulation are individualized.

In summary, if the patient in question had a mechanical mitral valve, I would continue to withhold warfarin for a period of seven to 10 days. I might then consider reintroducing anticoagulants; however, I would be likely to place the patient on low-dose low molecular weight heparin or unfractionated heparin rather than directly back on warfarin. If there was no evidence of additional bleeding after an additional period of one to two weeks, I might then gingerly reintroduce warfarin. Alternately, after seven to 10 days of warfarin withdrawal, the patient could be re-endoscoped to assess healing; if healing has not occurred, I would continue to withhold warfarin.

It is important to note that treatment recommendations in this setting are entirely based on anecdotal experience as there are little or no methodologically rigorous data on which to base treatment recommendations.

PA: Is there a role for a trial on heparin, instead of warfarin, because it has a shorter half-life? Can you discuss the difference between using regular heparin subcutaneously or infusion versus low molecular weight heparin?

MC: In patients at the highest risk of both bleeding and thrombosis (eg, a patient with a very recently inserted mitral valve), intravenous heparin is the anticoagulant of choice since it can be stopped and neutralized effectively. However, except in those patients requiring ongoing hospitalization, intravenous unfractionated heparin is impractical. Unfractionated heparin can be given subcutaneously in either prophylactic or therapeutic doses; traditionally, if used in therapeutic doses, heparin is monitored with a midinterval activated partial thromboplastin time, which reduces its utility in the outpatient setting. Low molecular weight heparin offers predictable, weightadjusted, unmonitored outpatient therapy when used in therapeutic doses; however, it is not completely neutralizable should bleeding occur and it may bioaccumulate in patients with impaired renal function.

Heparin or low molecular weight heparin does offer a potential bridge to full-dose anticoagulation; again, although not evidence-based, I will often times use lower doses of heparin or low molecular weight heparin while awaiting documentation of healing of gastrointestinal lesions or in the periprocedural period, when warfarin must be withheld.

PA: When do you advocate reversing anticoagulation rather than just stopping the medication, and how does this increase the risk of thrombotic events?

MC: There are two methods of reversal; the first is immediate reversal with plasma-derived clotting factors (most often in the form of fresh frozen plasma) and the second is the administration of vitamin K. Clotting factors should be administered to all patients with life-threatening bleeding; vitamin $\mathrm{K}$ should be simultaneously administered intravenously to ensure that 'rebound' anticoagulation does not occur as the transfused coagulation factors are consumed. Intravenous vitamin $\mathrm{K}$ will correct most patients' international normalized ratio (INR) values within $24 \mathrm{~h}$ to $36 \mathrm{~h}$ when given in doses of $2.5 \mathrm{mg}$ to $5.0 \mathrm{mg}$; this is an attractive agent if urgent but not emergent reversal is desired.

Acute correction of the INR is not known to increase the risk of clotting over and above the risk implicit in a normal INR; however, some reversal agents, such as FEIBA and recombinant factor VIla, likely do increase the risk of acute thrombosis. Therefore, plasma products should be reserved for patients with life-threatening bleeding.

PA: Clopidogrel is a relatively new medication with a long half-life. Can you tell us about the duration of antiplatelet effects? Does a patient ever need platelet infusions when bleeding on clopidogrel? Should clopidogrel be stopped for elective polypectomy?

MC: Plavix (clopidogrel) is a highly effective antiplatelet agent that produces its anticoagulant effect via active metabolites. Clopidogrel and its metabolites have a long-half life (approximately $12 \mathrm{~h}$ ) and thus, prolonged and irreversible platelet inhibition occurs in patients who have received clopidogrel. Reports of detectable levels of clopidogrel or its metabolites have been reported for more than a week after a single dose. Platelet transfusion may slow or stop bleeding in patients with life-threatening or intracerebral bleeding; although clopidogrel metabolites will impair transfused platelets, this impairment will not occur immediately and thus, transfused platelets may provide temporary hemostasis. Discontinuing any antithrombotic drug will increase the risk of a clotting complication; decisions about discontinuation should be individualized. Since polypectomy is associated with bleeding at the stalk site where the polyp is transected, and is more likely to occur with larger, pedunculated polyps, discontinuation of all antithrombotics should be considered. However, if the person had a recent coronary stent, for example, I might consider continuing deferring the polypectomy until a four to six week period had passed.

PA: We have been discussing mostly atherosclerosis and anticoagulants. Let's consider the scenario of a patient with a recent pulmonary embolism or deep vein thrombosis (DVT) in the leg who is having GI bleeding on anticoagulants (heparin or warfarin). How quickly do you move to umbrella therapy in this situation?

MC: If the person had a recent DVT or pulmonary embolism (usually defined as within the past month), they are at high risk for symptomatic recurrence, and if the bleeding is major or life-threatening, immediate inferior vena cava filter insertion should be arranged. Temporary filters are very handy in this situation. If the source of bleeding is likely correctable, a temporary filter can be inserted for up to two weeks (and moved, if required), allowing heparin/warfarin to be withheld for extended periods. Permanent inferior vena cava filters increase the risk of recurrent DVT if left in situ; therefore, a permanent filter should only be inserted if clearly justified by the clinical situation.

PA: How do you approach the patient with portal hypertension and esophageal varices who may have an indication for anticoagulation? For example, I have a young woman with a portal vein thrombosis and esophageal varices who was found to have a positive anticardiolipin antibody (ACA) and lifelong anticoagulation has been recommended. Banding of esophageal varices in any patient can cause bleeding either during the procedure or five to seven days later when the bands fall off. Any time we stop anticoagulants to perform GI procedures, we worry that the patient may have another blood clot leading to serious morbidity or mortality. Any advice on this topic?

MC: This is a very difficult issue, and is often times compounded by some degree of underlying 'autoanticoagulation' due to hepatic dysfunction. Most simply, there is no correct answer. Anticoagulants will increase the risk of bleeding and will make 
bleeding that does occur worse. If the patient has a very high risk of thrombosis, the risk of anticoagulation might be justifiable, but in many circumstances, the enhanced risk of bleeding 'trumps' the risk of clots. For example, most patients with atrial fibrillation will have a risk of thrombosis far lower than their risk of anticoagulant-related hemorrhage. Large randomized trials of warfarin have proven that lower intensity warfarin (target INR 1.5 to 2.0) is less effective and causes as much bleeding as usual intensity warfarin - it should not be used in this setting.

In this particular case, I would carefully examine the indication for anticoagulation and consider discontinuing warfarin. I would question the clinical significance of a positive ACA in the setting of a patient with chronic liver disease and portal vein thrombosis, since many patients have asymptomatic ACA and in the absence of prior thrombosis, do not have an increased risk for first thrombosis.

PA: Do you have any experience with using heparin to provoke a GI bleed to identify a source in a nuclear red blood cell scan or angiogram?

MC: We often refer to this as the anticoagulant stress test. Although intrinsically appealing, this strategy could be associated with uncontrollable bleeding and I would strongly recommend against using it. Furthermore, there are no well-designed studies that have assessed the diagnostic yield and, most importantly, the safety of this approach.

ACKNOWLEDGEMENTS: Mark Crowther acknowledges the advice of James Douketis. 


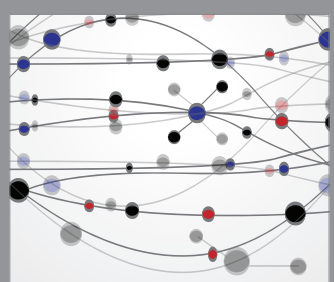

The Scientific World Journal
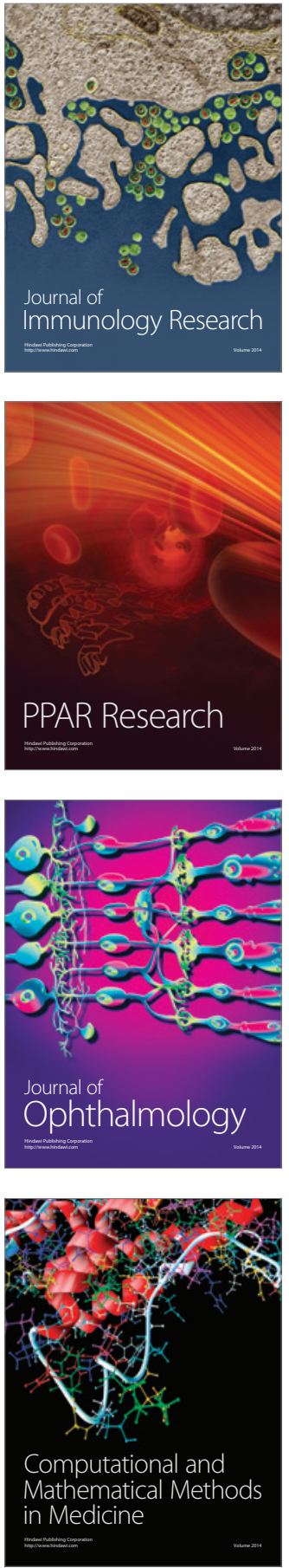

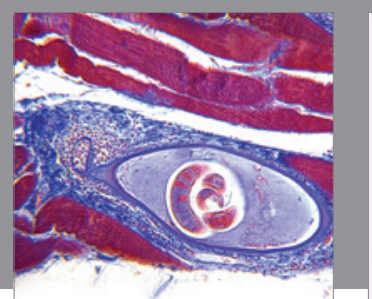

Gastroenterology Research and Practice

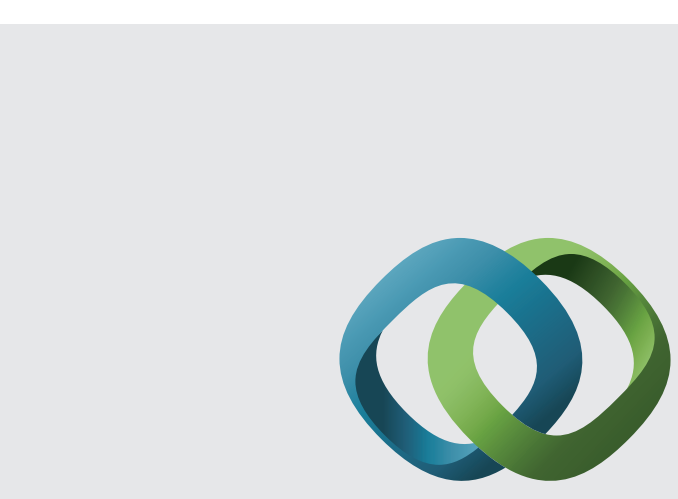

\section{Hindawi}

Submit your manuscripts at

http://www.hindawi.com
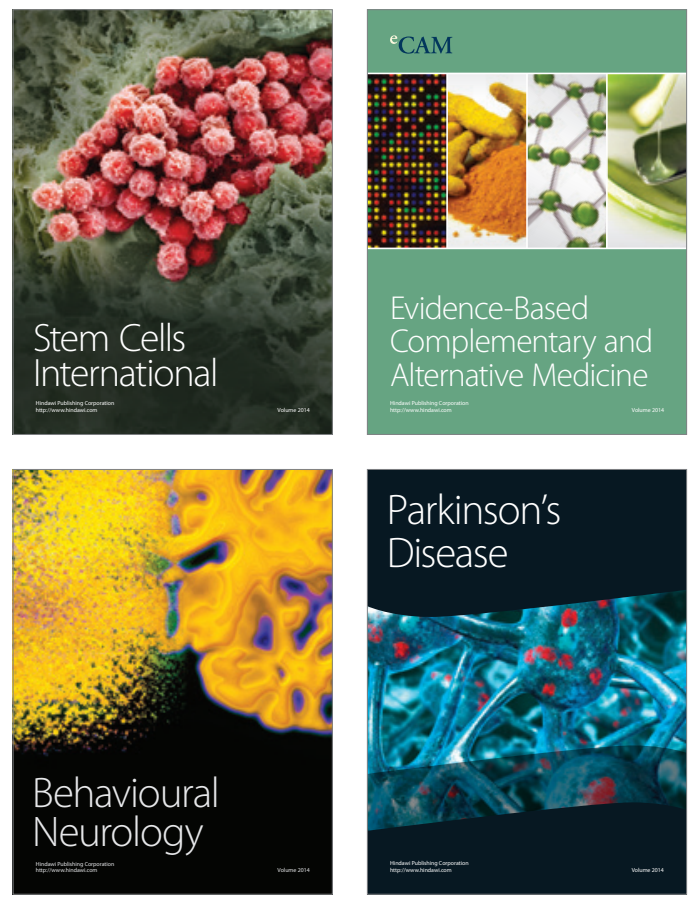
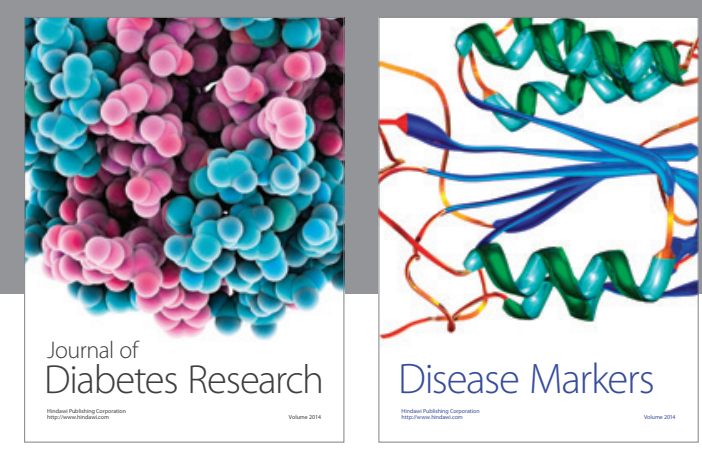

Disease Markers
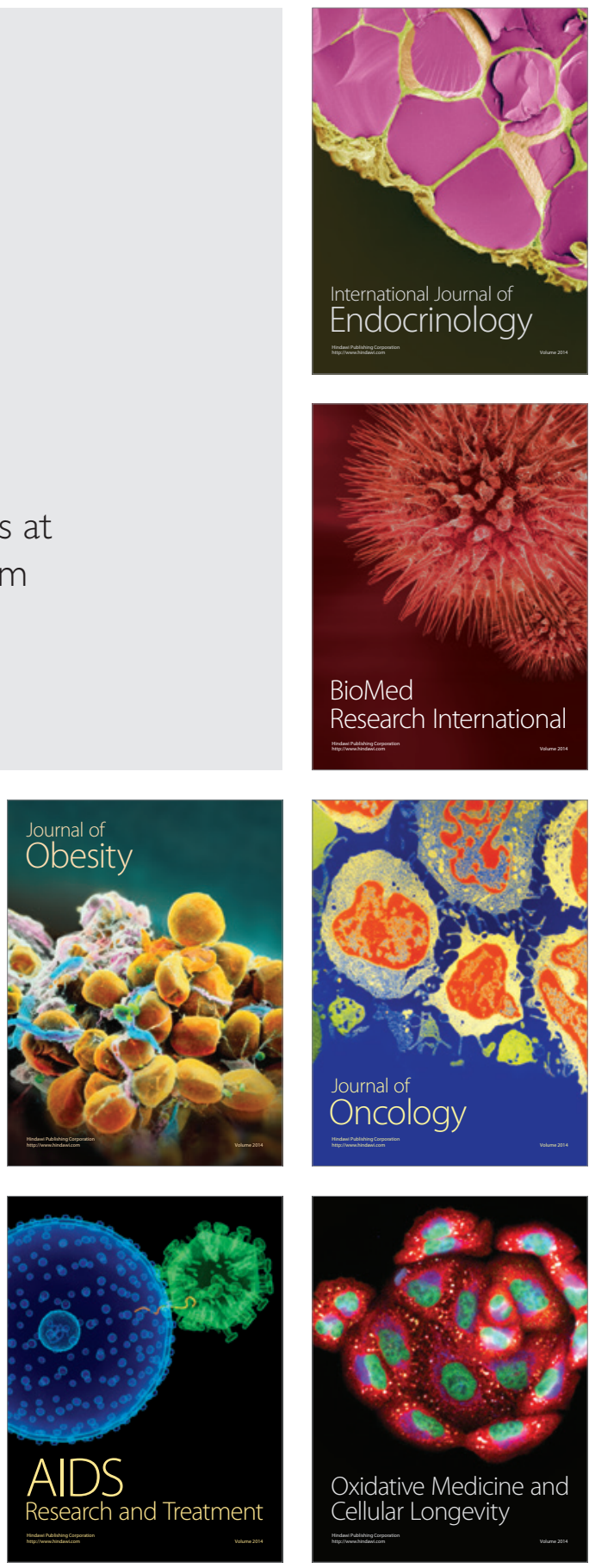